Supporting Information

\title{
Fingerprinting the Ammonia Synthesis Pathway Using Spatiotemporal Electrostatic Potential Distribution of Intermediates
}

\author{
Jialu Li, ${ }^{\ddagger}$ Xiaochen Shen, ${ }^{\ddagger}$ Yanbo Pan, Zhenmeng Peng* \\ Department of Chemical, Biomolecular, and Corrosion Engineering, The University of Akron, \\ Akron, Ohio 44325, United States
}




\section{Content}

\section{Supplementary Figures}

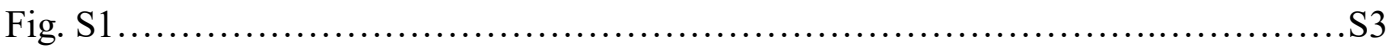

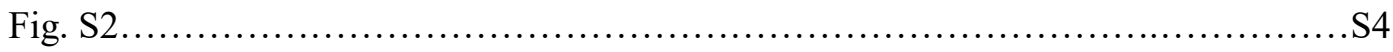

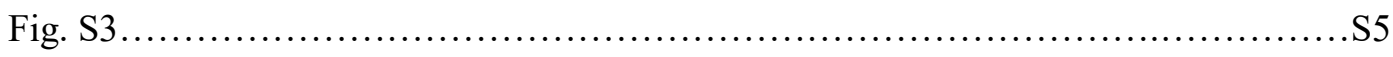

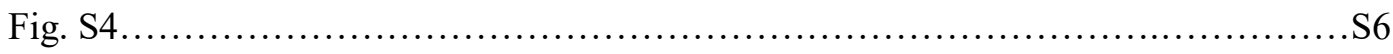

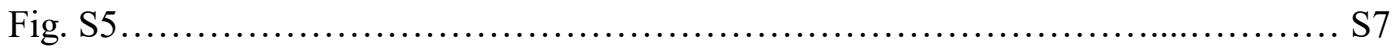

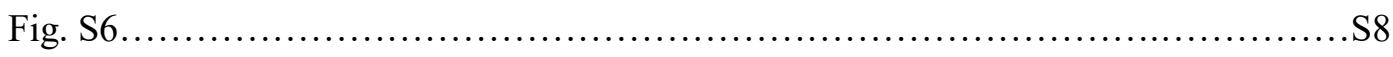

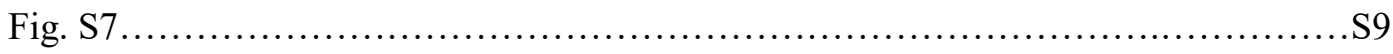

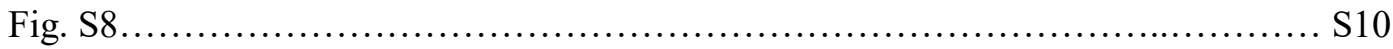

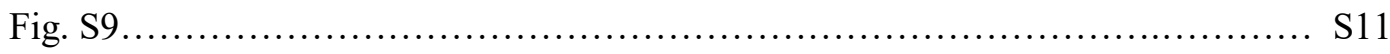

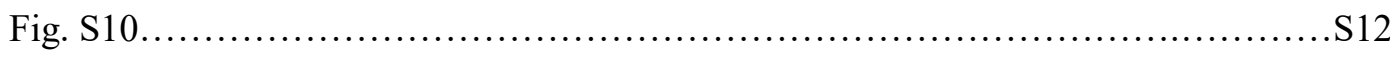

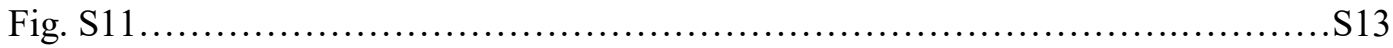

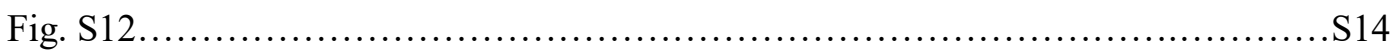

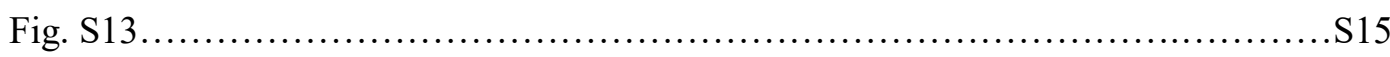

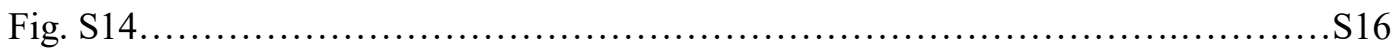

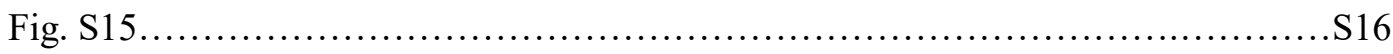

\section{Supplementary Tables}

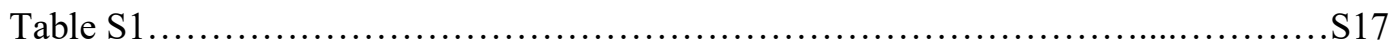

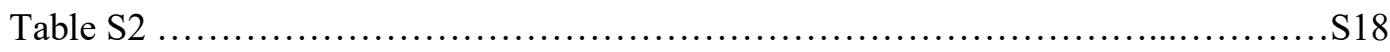

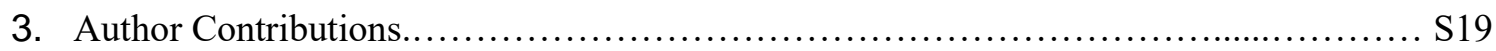




\section{Supplementary Figures}

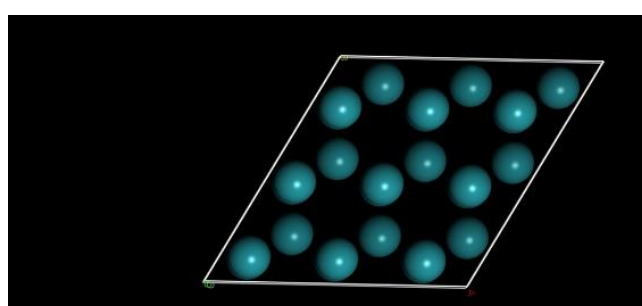

top view

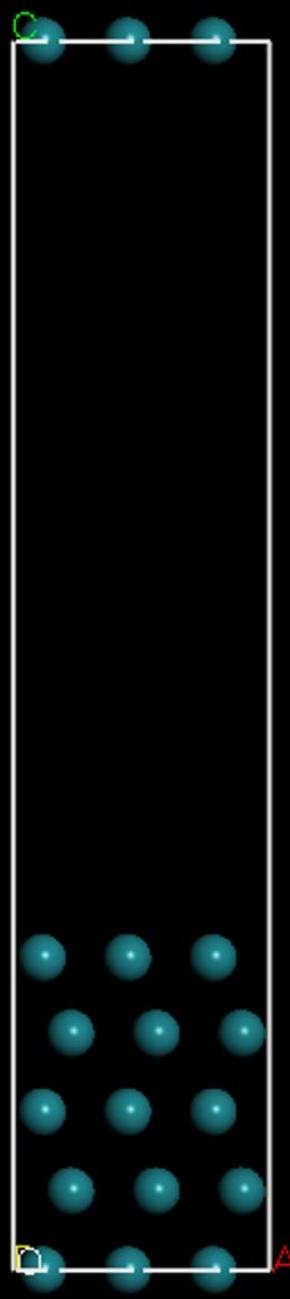

front view

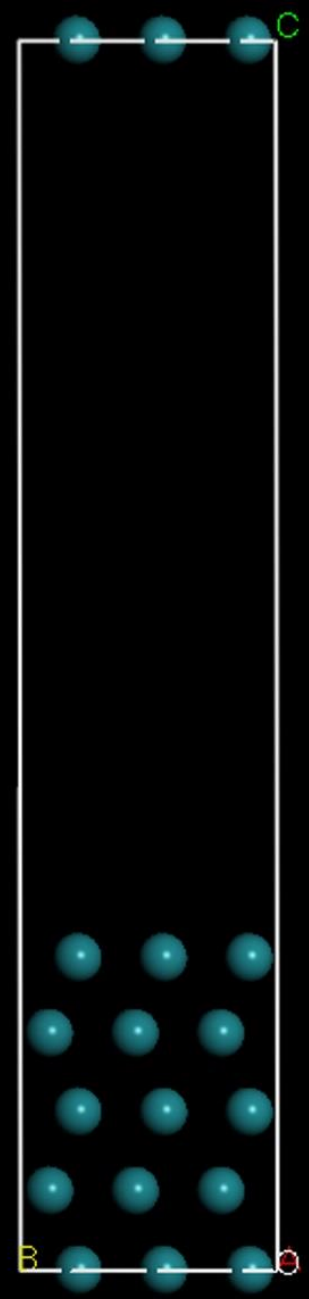

side view

Figure S1. Three standard views of the optimized $\mathrm{Ru}$ model ( $\mathrm{Ru}$ atom-green) 


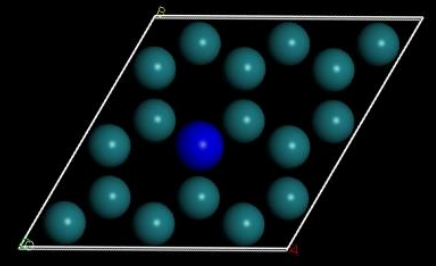

top view
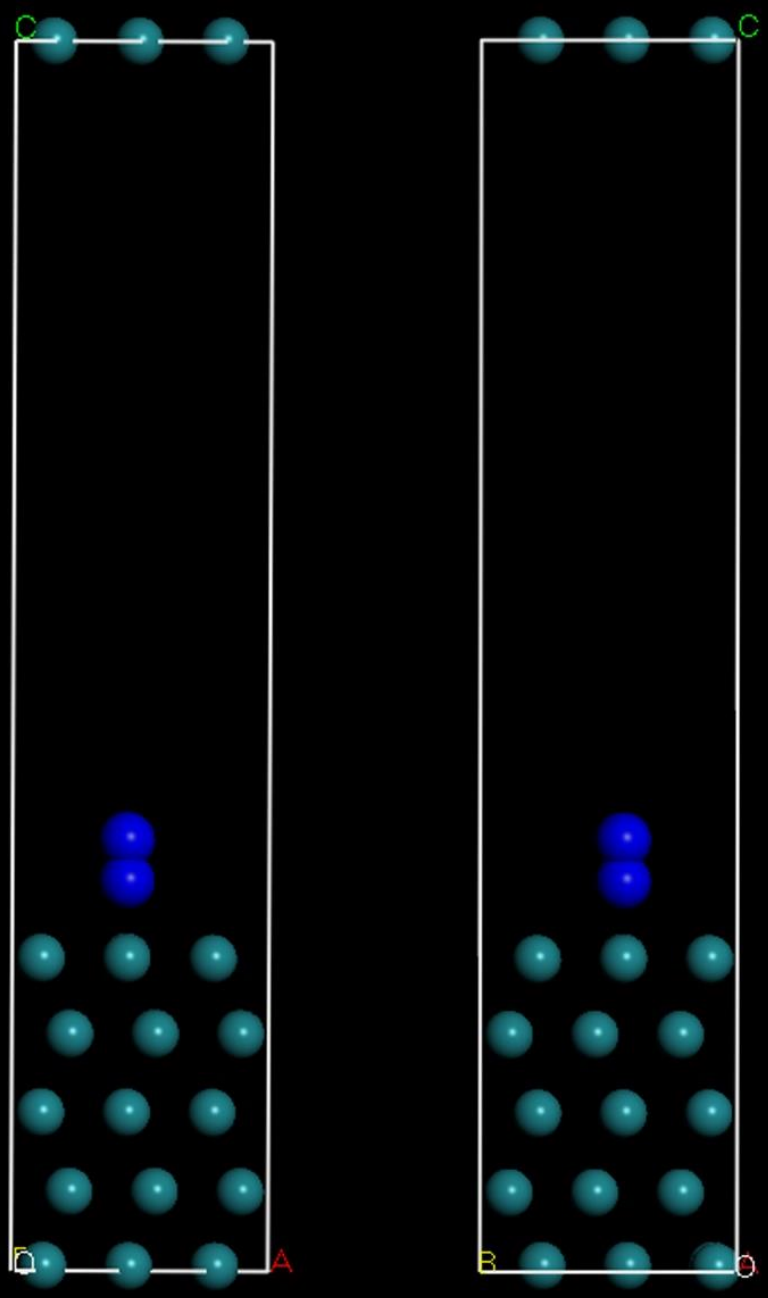

front view side view

Figure S2. Three standard views of *N-N model (N atom-blue) 


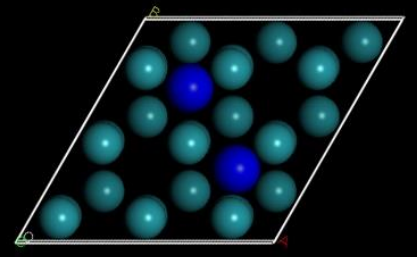

\section{top view}


front view

\section{side view}

Figure S3. Three standard views of $2 * \mathrm{~N}$ model 




\section{top view}

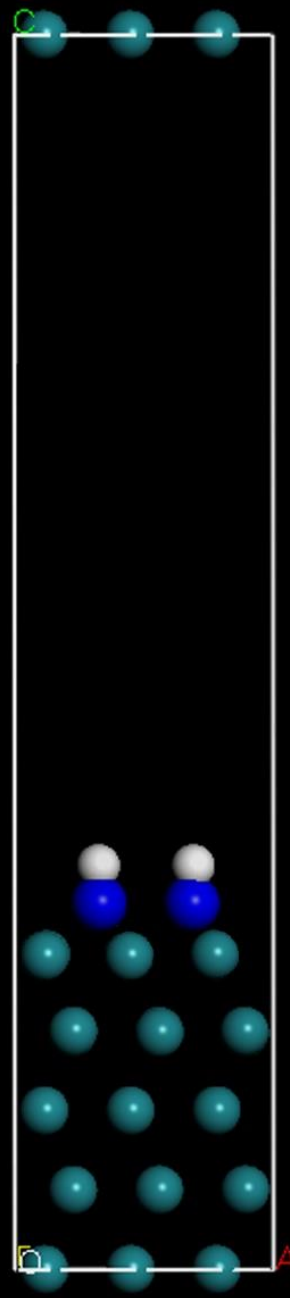

front view

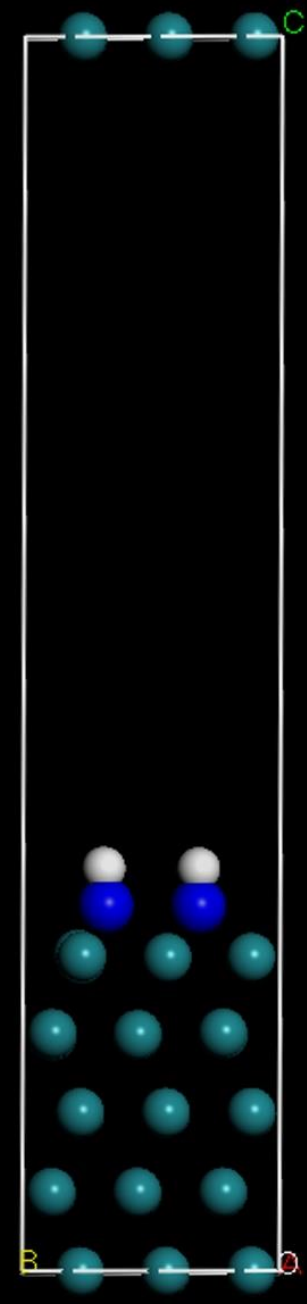

side view

Figure S4. Three standard views of $2 * \mathrm{NH}$ model ( $\mathrm{H}$ atom-white) 


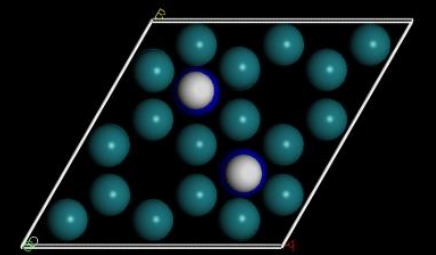

\section{top view}

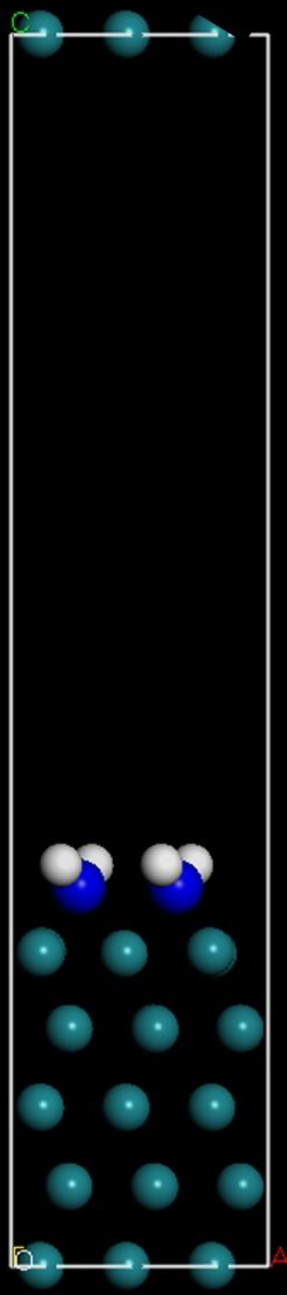

front view

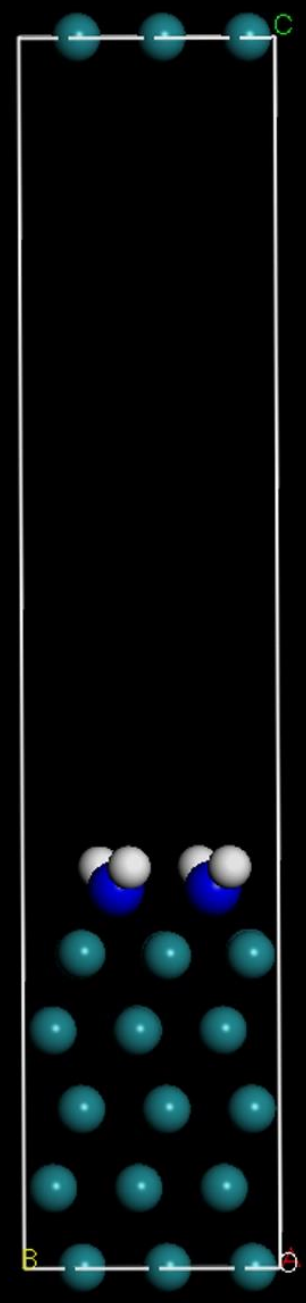

side view

Figure S5. Three standard views of $2 * \mathrm{NH}_{2}$ model 


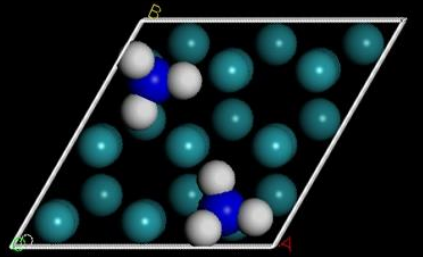

top view

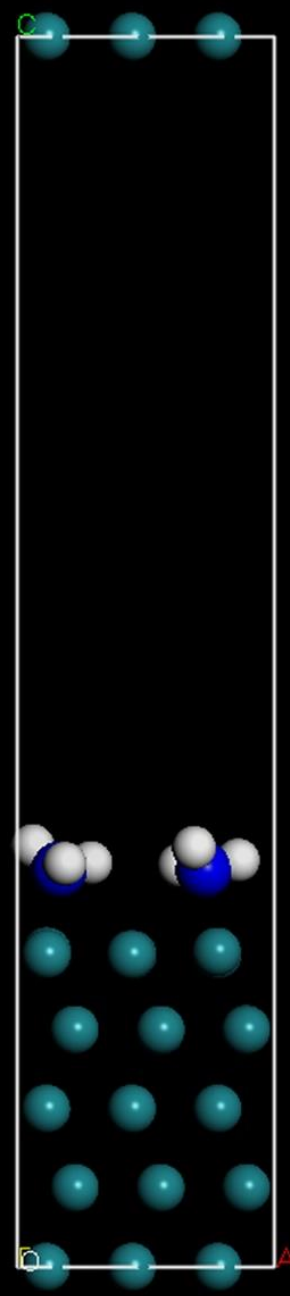

front view

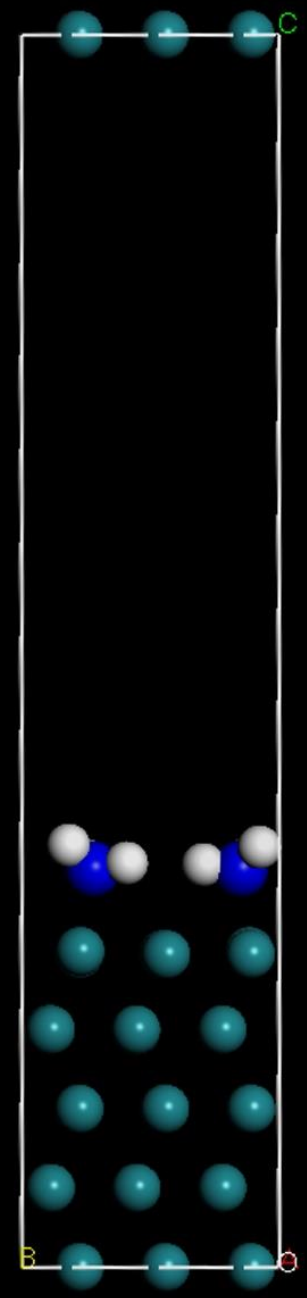

side view

Figure S6. Three standard views of $2 * \mathrm{NH}_{3}$ model 




\section{top view}
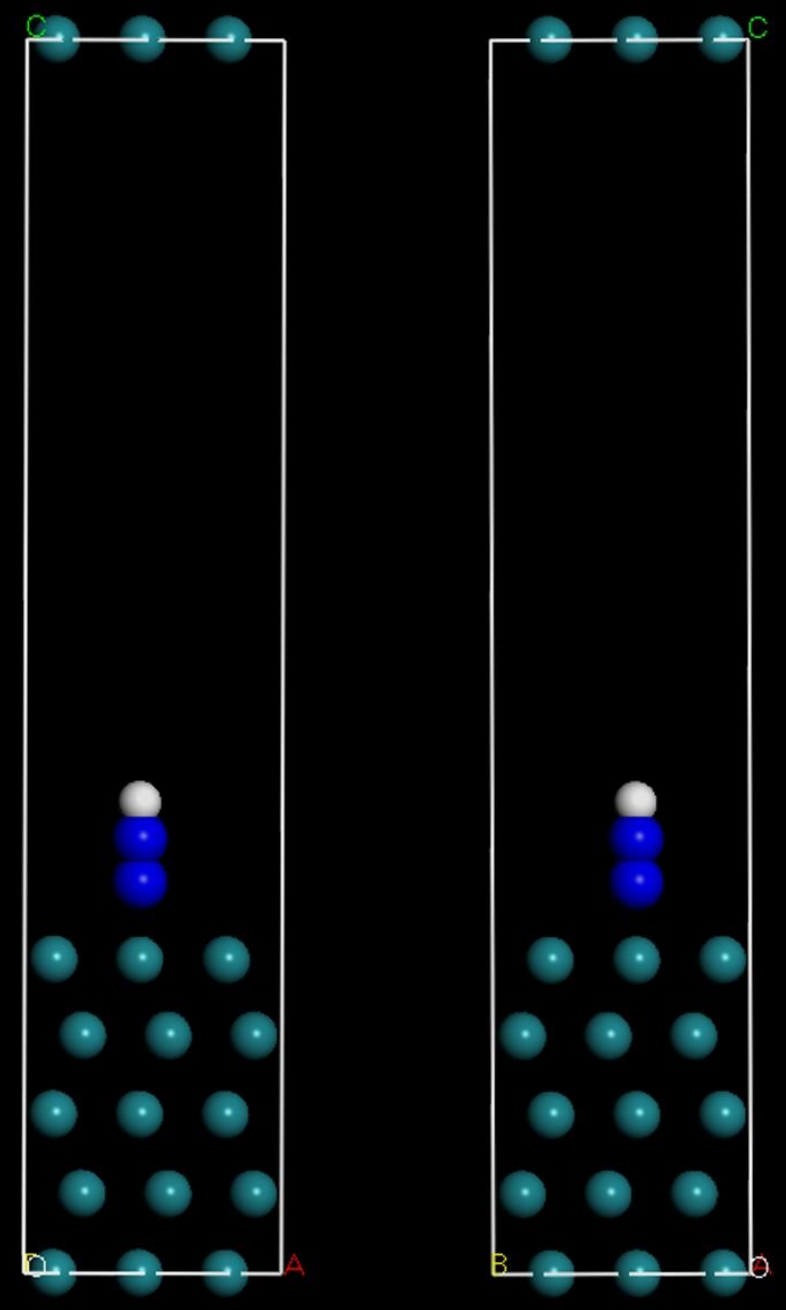

front view

\section{side view}

Figure S7. Three standard views of $* \mathrm{~N}-\mathrm{NH}$ model 




\section{top view}
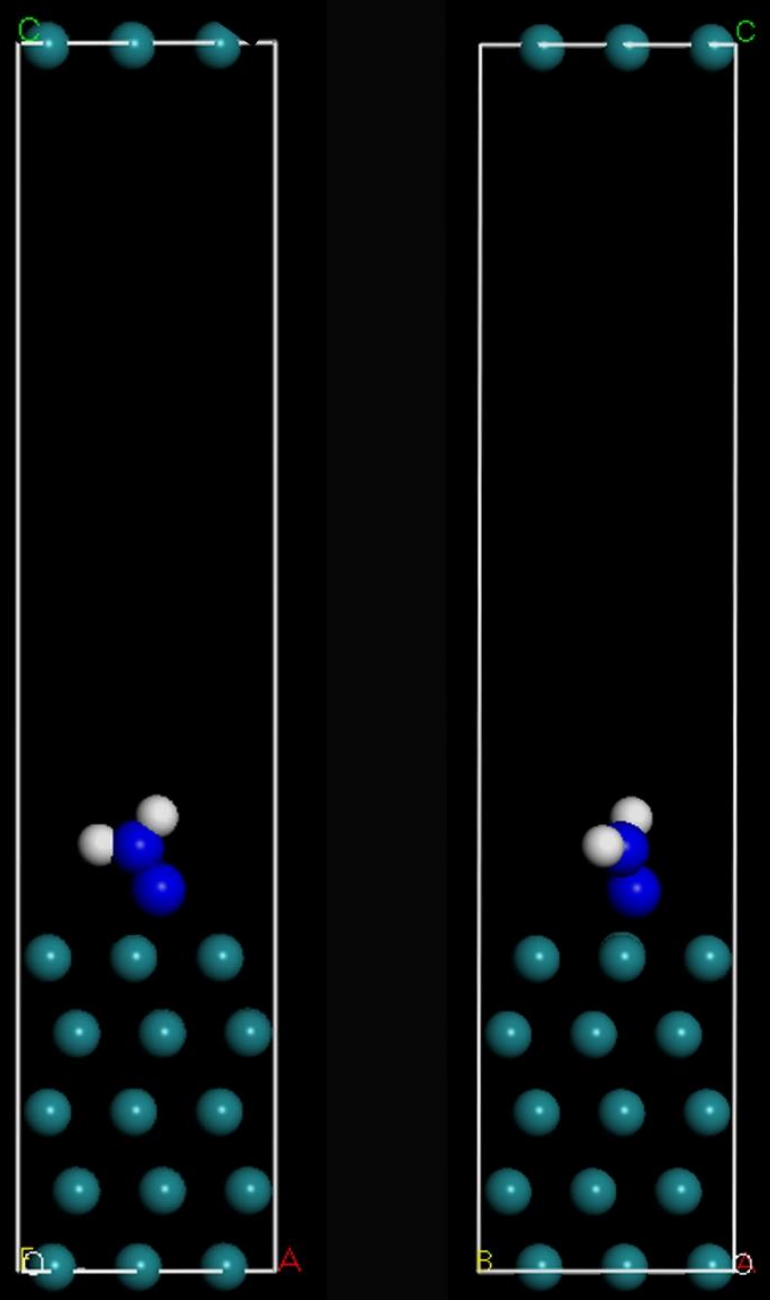

front view side view

Figure S8. Three standard views of $* \mathrm{~N}-\mathrm{NH}_{2}$ model 


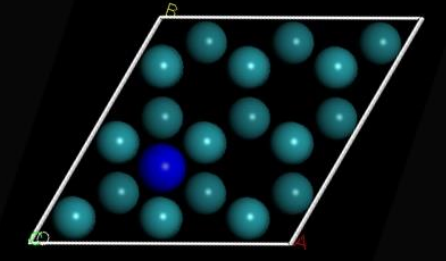

\section{top view}

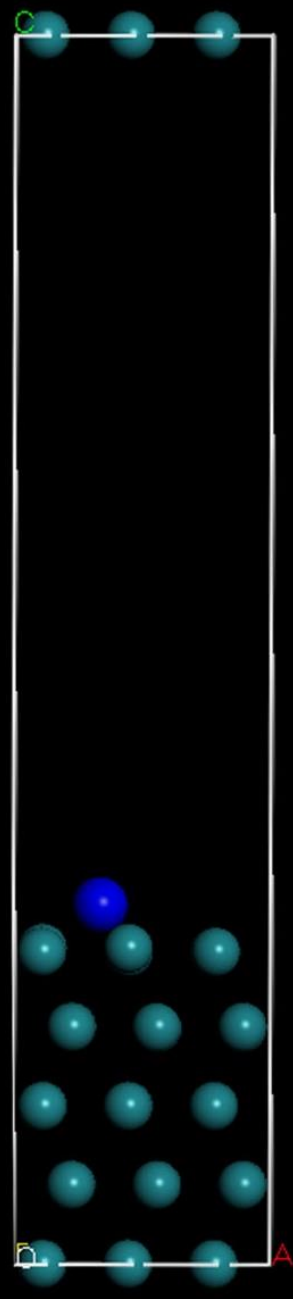

front view

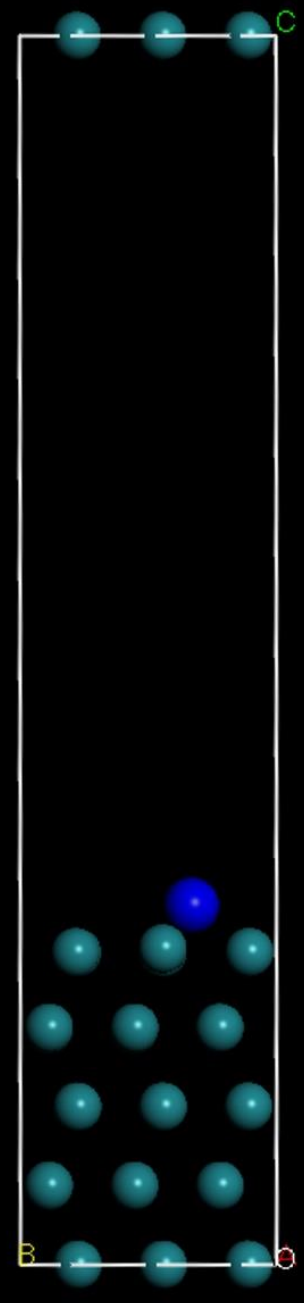

side view

Figure S9. Three standard views of $* \mathrm{~N}$ model 


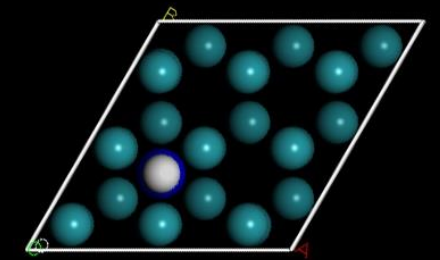

\section{top view}



front view

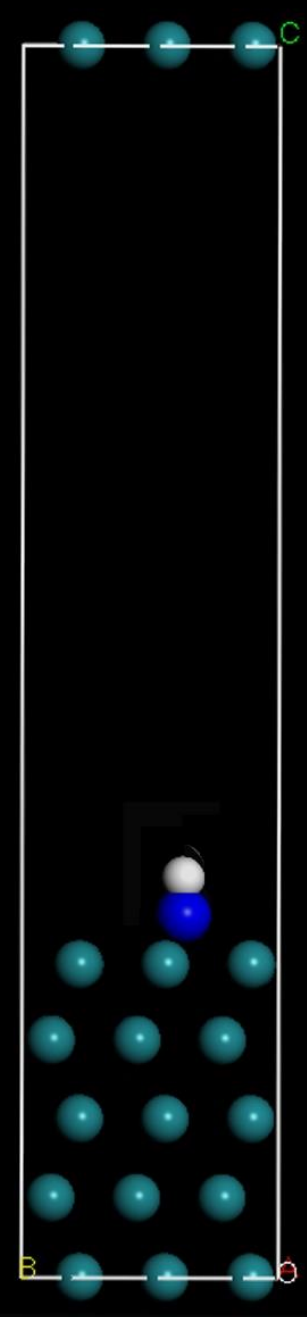

side view

Figure S10. Three standard views of $* \mathrm{NH}$ model 




\section{top view}
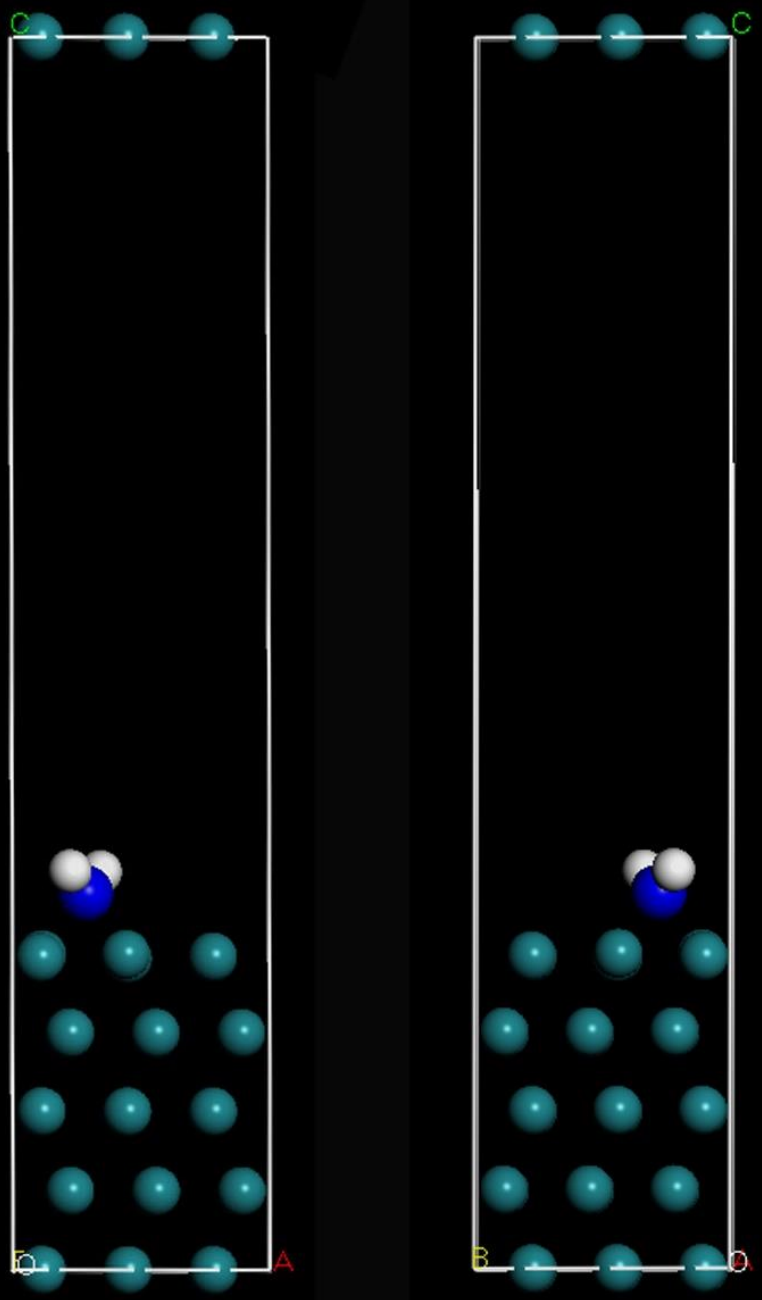

front view

\section{side view}

Figure S11. Three standard views of $* \mathrm{NH}_{2}$ model 


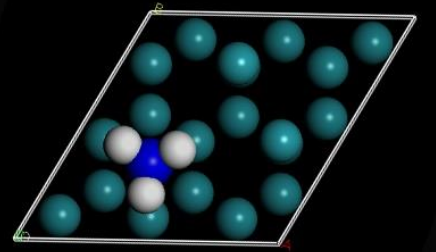

\section{top view}

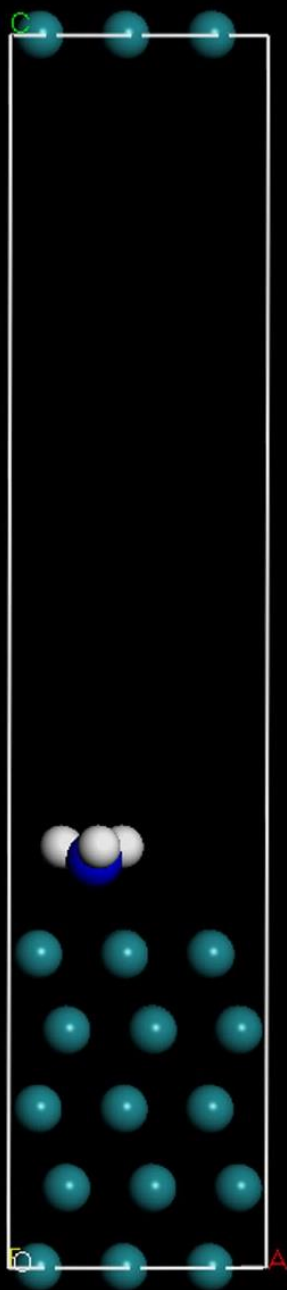

front view



side view

Figure S12. Three standard views of $* \mathrm{NH}_{3}$ model 


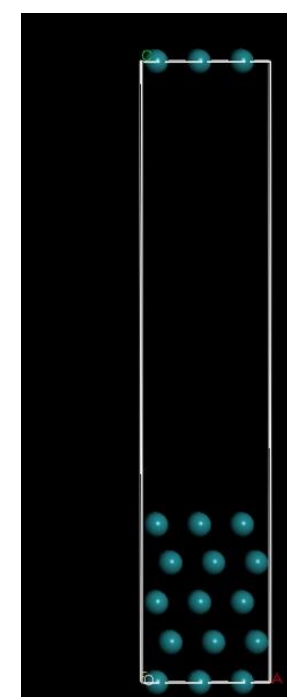

optimized Ru

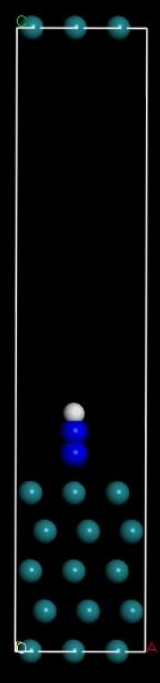

*N-NH

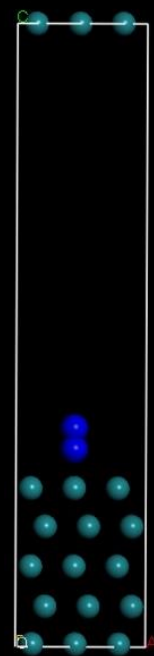

*N-N

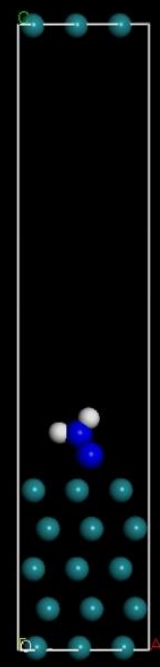

*N-NH${ }_{2}$

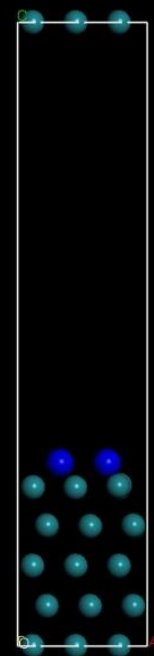

$2 * \mathrm{~N}$

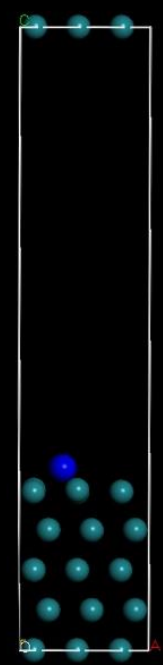

$* \mathrm{~N}$

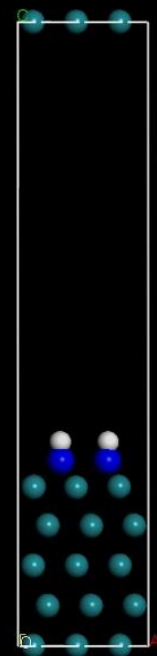

$2 * \mathrm{NH}$

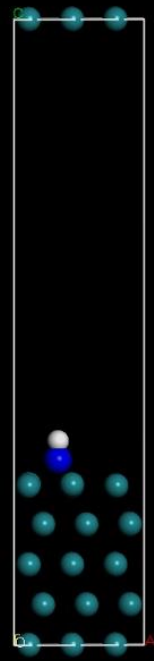

$* \mathrm{NH}$

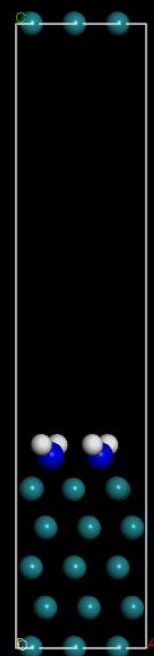

$2 * \mathrm{NH}_{2}$



$* \mathrm{NH}_{2}$

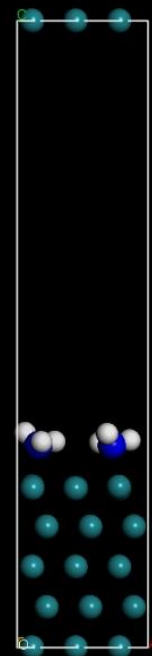

$2 * \mathrm{NH}_{3}$

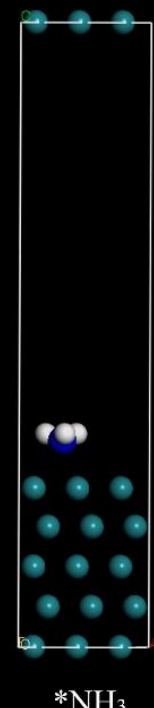

Figure S13. Summary of intermediate models in the simulation (Ru atom-green; $\mathrm{N}$ atom-blue; $\mathrm{H}$ atom-white) 


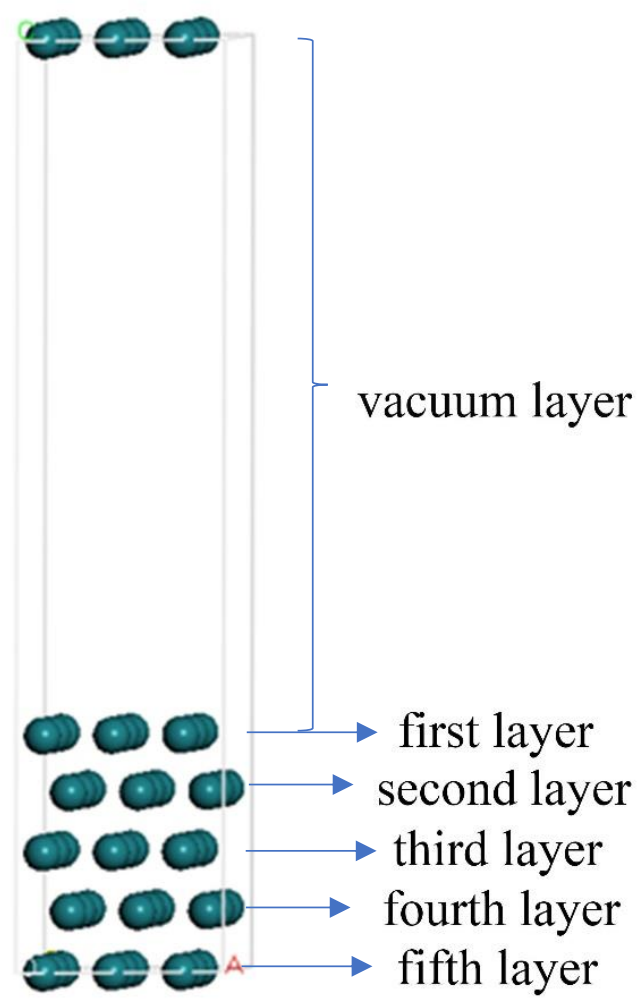

Figure S14. Ru (001) slab model in the simulation

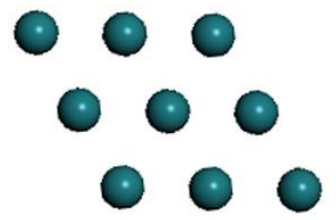

Figure S15. The First layer of the $\mathrm{Ru}(001)$ 


\section{Supplementary Tables}

\begin{tabular}{|c|c|}
\hline \multicolumn{2}{|c|}{ Dissociative mechanism } \\
\hline Reaction species & Relative energy (eV) \\
\hline $\mathrm{N}_{2}$ & 0 \\
\hline$* \mathrm{~N}-\mathrm{N}$ & -0.5355 \\
\hline $2 * \mathrm{~N}$ & -0.2488 \\
\hline $2 * \mathrm{NH}$ & -1.9928 \\
\hline $2 * \mathrm{NH}_{2}$ & -2.5428 \\
\hline $2 * \mathrm{NH}_{3}$ & -3.2835 \\
\hline $\mathrm{NH} 3$ & -2.1472 \\
\hline & Associative mechanism \\
\hline Reaction species & Relative energy (eV) \\
\hline $\mathrm{N} 2$ & 0 \\
\hline$* \mathrm{~N}-\mathrm{N}$ & -0.5355 \\
\hline$* \mathrm{~N}-\mathrm{NH}$ & 0.9543 \\
\hline$* \mathrm{~N}-\mathrm{NH} 2$ & 0.3075 \\
\hline$* \mathrm{~N}$ & -0.2483 \\
\hline$* \mathrm{NH}$ & -0.3769 \\
\hline$* \mathrm{NH} 2$ & -1.7439 \\
\hline$* \mathrm{NH} 3$ & -2.9597 \\
\hline $\mathrm{NH} 3$ & -2.1472 \\
\hline
\end{tabular}

Table S1. Summary of DFT calculation results

The adsorption energy of $E_{\text {ads }}$ was described as:

$E_{\text {ads }}=E_{\text {total }}-E_{\text {slab }}-E_{\text {adsorbate }}$

where $E_{\text {total }}$ is the total energy of the relaxed adsorbate adsorbed on the slab, $E_{\text {slab }}$ is the energy of the clean slab alone, $E_{\text {adsorbate }}$ is the energy of free adsorbate alone. 


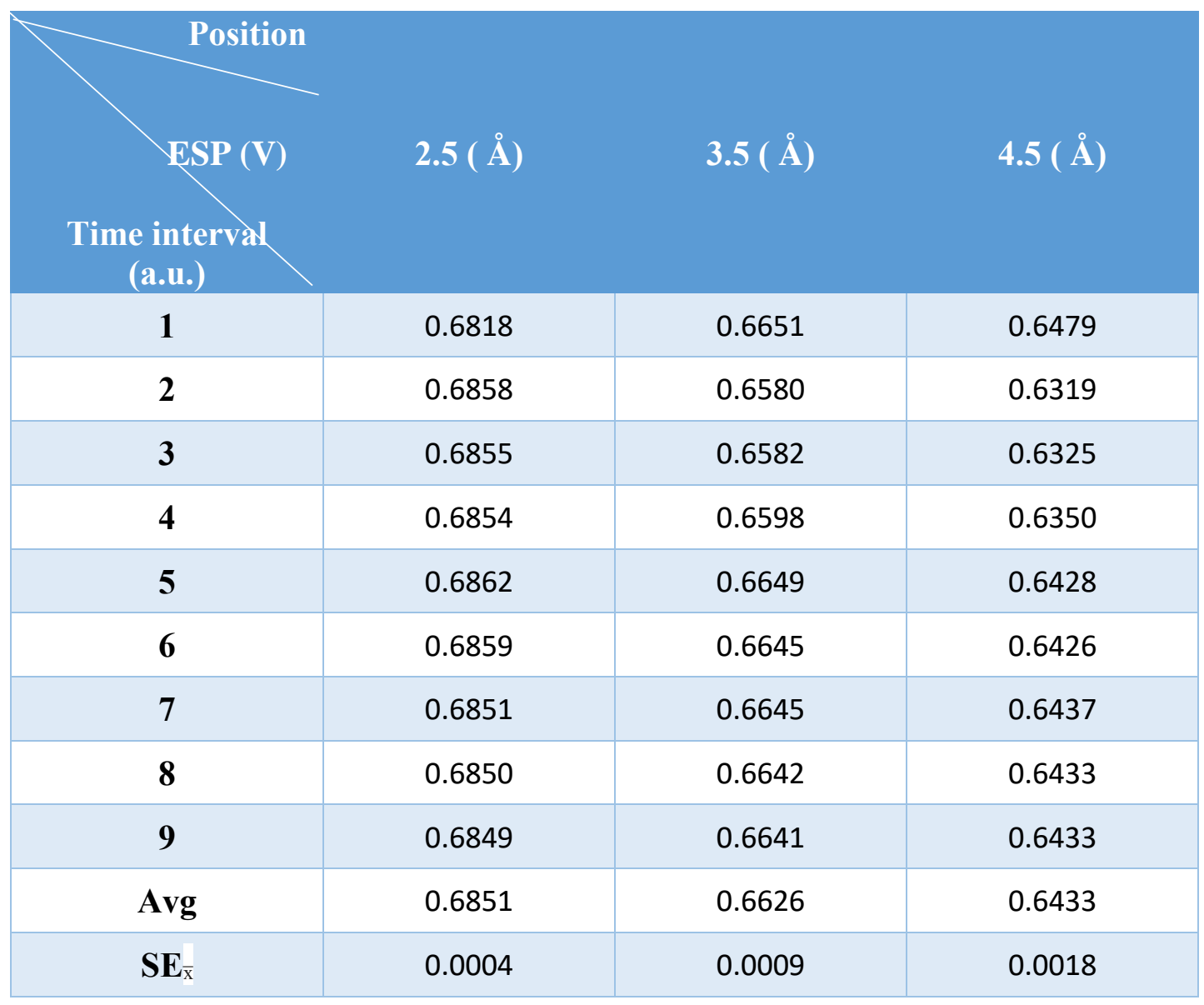

Table S2. Summary of the simulated ESP values of $* \mathrm{~N}-\mathrm{NH}_{2}$ 


\section{Author Contributions}

₹ These authors contributed equally. All authors have given approval to the final version of the manuscript. 